\title{
Geometry and reactor synthesis: maximizing conversion of the ethyl acetate process
}

\author{
N. Asiedu • D. Hildebrandt $\cdot$ D. Glasser
}

Received: 21 June 2014 / Accepted: 4 February 2015/Published online: 25 February 2015

(c) The Author(s) 2015. This article is published with open access at Springerlink.com

\begin{abstract}
This paper describes how experimentally generated results were used to optimize conversion of a process using adiabatic batch reactor systems. The process used in the experiment was exothermic reversible process. The experiments were conducted using Dewar Thermosflask operating under adiabatic conditions. Equilibrium conversions were determined from temperature-time information. Temperatures were determined using negative temperature coefficient thermistor. For a single batch process, the equilibrium conversion determined experimentally was shown to be 0.55 and 0.21 with respect to acetic acid using two initial temperatures of $283 \mathrm{~K}$ and $295 \mathrm{~K}$, respectively. It is shown by a simple geometrical approach that without the knowledge of the kinetics of the process, by increasing the number of reactors and considering internal cooling systems, the reaction equilibrium lines were crossed and conversion improved significantly. The paper also shows that one can attain the maximum possible conversion of 0.72 , thus increasing equilibrium conversions by $31 \%$ by adding a single adiabatic reactor to the single-stage adiabatic reactor by this geometrical technique, and hence proposes the optimal reactor
\end{abstract}

\footnotetext{
N. Asiedu ( $\square)$

Department of Chemical Engineering, College of Engineering, Kwame Nkrumah University of Science and Technology, University post office, Kumasi, Ghana

e-mail: nasiedusoe@yahoo.co.uk

D. Hildebrandt · D. Glasser

University of South Africa, 5th Floor, Pha-Pha Building, Cnr.

Pioneer and Christian De Wet Road, Private Bag X6,

Florida, Johannesburg 1710, South Africa

e-mail: diane.hildebrandt@outlook.com

D. Glasser

e-mail: David.Glasser007@gmail.com
}

configuration with interstage cooling system to achieve this optimal conversion.

Keywords Conversion - Equilibrium line $\cdot$ Adiabatic batch reactors

$\begin{array}{ll}\text { List of symbols } \\ \Delta H_{\mathrm{rxn}} & \text { Heat of reaction }(\mathrm{KJ} / \mathrm{mol}) \\ \Delta T_{\mathrm{ad})} & \text { Adiabatic temperature change }(\mathrm{K}) \\ \Delta \varepsilon & \text { Extent of reaction } \\ C_{\mathrm{P}} & \text { Constant heat capacity of the reaction mixture } \\ & \quad \text { KJ } / \mathrm{mol} \mathrm{K}) \\ m & \text { Mass of the reaction mixture }(\mathrm{Kg}) \\ t & \text { Time }(\mathrm{s}) \\ T_{0} & \text { Basis temperature }(\mathrm{K}) \\ T & \text { Reactor temperature }(\mathrm{K}) \\ T_{\mathrm{S}} & \text { Steady-state temperature }(\mathrm{K}) \\ U & \text { Heat transfer coefficient }\left(\mathrm{J} / \mathrm{m}^{2} \mathrm{~s} \mathrm{~K}\right) \\ x & \text { Conversion }\end{array}$

\section{Introduction}

Maximization of process conversion of reactants to products has always been one of the main concerns in process engineering. In an exothermic reversible reaction occurring in an adiabatic batch reactor, the maximum conversion (equilibrium conversion) is achieved when the process reaches equilibrium, which is the situation where the rates of forward and backward reactions are equal in a typical equilibriumlimited process like esterification reactions. Thus, the concept of equilibrium therefore becomes a barrier which cannot be overcome in principle with a single adiabatic batch reactor. For adiabatic exothermic reversible reaction, equilibrium 
conversion is a function of temperature; the higher the initial feed temperature, the lower is the equilibrium conversion. Thus for a higher equilibrium conversion, the size of the reactor required for the duty becomes a critical issue. At lower temperatures, the reaction rates are smaller; this implies that for a cooler feed a bigger reactor is required to achieve a given or desired conversion. Hence in the optimization problem is to find reversible process, a typical optimization problem is to find a way to obtain high conversion and at the same time minimize cost which is directly related to the size of the reactor structure. Industrially, direct means of cooling down reaction mixtures are employed [1]. Another practice in industrial settings is to externally reduce the temperature of the reaction mixtures [2,3]. By manipulating heating and cooling in using internal streams in exothermic reversible process, it is possible to maximize the process in terms of conversion and reactor size. In this work the focus is to maximize conversion of the process without taking into consideration the kinetics and the cost effect associated with the synthesis. This result can be further investigated when cost implications are taken into consideration.

\section{The mathematical model of the reacting system- thermos flask}

Given an adiabatic batch reactor (thermos flask), the mathematical model is made up of a set of differential equations resulting from the mass and energy balances referred only to the reaction mixture because there is no heat transfer.

The stoichiometry of the reactions studied is given below:

$$
\begin{gathered}
\mathrm{CH}_{3} \mathrm{COOH}+\mathrm{CH}_{3} \mathrm{CH}_{2} \mathrm{OH} \leftrightarrow \mathrm{CH}_{3} \mathrm{COOCH}_{2} \mathrm{CH}_{3} \\
+\mathrm{H}_{2} \mathrm{O}, \Delta H_{\mathrm{rxn}}(298 \mathrm{~K})=-6.12 \mathrm{~kJ} / \mathrm{mol} .
\end{gathered}
$$

For a constant-volume batch reactor one can write:

$-r_{\mathrm{A}}=\frac{1}{V} \frac{\mathrm{d} N_{\mathrm{A}}}{\mathrm{d} t}=-\frac{\mathrm{d} C_{\mathrm{A}}}{\mathrm{d} t}$.

The energy balance equation can be established as:

Heat generated $=$ Heat absorbed by reactor contents

$$
+ \text { heat transferred through reactor walls, }
$$

$(-\Delta H)\left(-r_{\mathrm{A}}\right) V \mathrm{~d} t+Q_{\text {stirrer }} \mathrm{d} t=m C_{\mathrm{P}} \mathrm{d} T+U A(\Delta T) \mathrm{d} t$,

Given

$\left(-r_{\mathrm{A}}\right)=\frac{\mathrm{d} \varepsilon}{\mathrm{d} t}$

where $(\varepsilon)$ is the extent of reaction. We can rearrange Eq. (4) to give Eq. (6) below:
$U A(\Delta T) \mathrm{d} T+m C_{\mathrm{P}} \mathrm{d} T=\frac{(-\Delta H) \mathrm{d} \varepsilon}{\mathrm{d} t}$.

Assume that heat given by the stirrer speed $\left(Q_{\text {strirrer }}\right)$ is negligible. Integrating Eq. (6) gives

$\left(T-T_{0}\right)+\frac{U A}{m C_{\mathrm{P}}} \int_{0}^{\infty}\left(T-T_{0}\right) \mathrm{d} t=\frac{(-\Delta H)}{m C_{\mathrm{P}}} \Delta \varepsilon$.

The LSH of Eq. (7) can be used to correct experimental data to adiabatic conditions.

It is assumed that the heat of the reaction is independent of temperature; hence, correcting the experimental data the adiabatic temperature rise parameter $\left(\Delta T_{\mathrm{ad}}\right)$ can be obtained from the experimental result for the process. This adiabatic temperature rise is equal to the RHS of Eq. (7) or we can write:

$\Delta T_{\mathrm{ad}}=\frac{(-\Delta H) \varepsilon}{m C_{\mathrm{P}}}$.

From Eq. (8), one can easily show that the energy balance equation of an adiabatic batch reactor reduces to a linear form given by Eq. (9) as:

$T=T_{0}+\Delta T_{\mathrm{ad}} \varepsilon$.

Theoretically, the adiabatic temperature rise is by definition obtained when the extent of reaction $(\varepsilon)=1$ or conversion $(x)=1$, with respect to the reactant of interest and its value can be computed in advance from the initial conditions (temperature and heat capacities) of the reacting species. Equation (9) also allows one to find the extent of reaction and or conversion at any instant under adiabatic conditions by using only one measure of temperature. Then from the initial concentrations of the reactants, the concentrations of the products can be monitored at any time in the reactor.

\section{Experimental descriptions}

The experimental setup as shown in Fig. 1 was used in all the reactions studied. The adiabatic batch reactor used in the experiments is $18 / 8$ stainless steel thermos flask of total volume $500 \mathrm{~mL}$ equipped with a removable magnetic stirrer. The flask is provided with a negative temperature coefficient thermistor connected online with a data-logging system. The signal from the sensor (thermistor) is fed to a measuring and a control unit amplifier and a power interface. The acquisition units are connected to a data processor, a process control engineering support data management system. The data acquisition system called Clarity has the following part numbers: C50 Clarity Chromatography SW, single instrument, $3 \times 55$ Clarity Add-on instrument SW and 194 INT9 quad channel A/D converter card. 


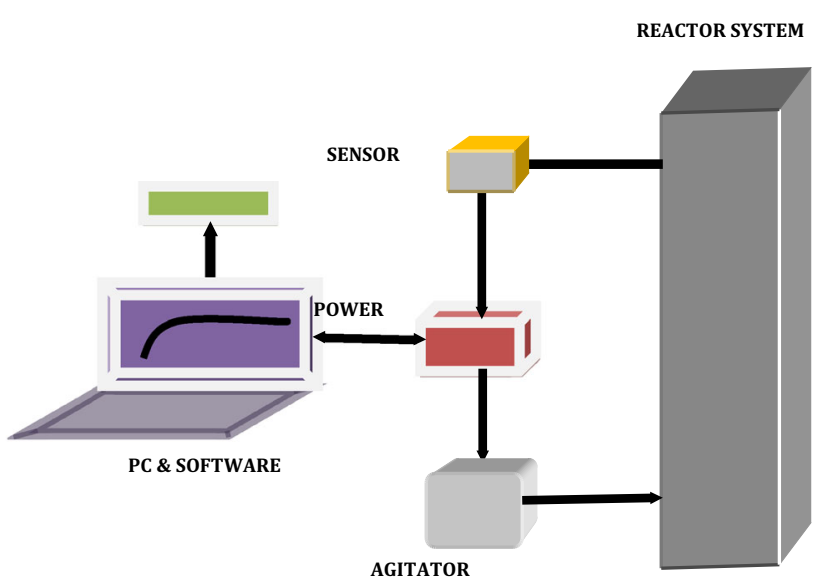

Fig. 1 Block diagram of the experimental setup

The hardware is INT9 PCI A/D 24 bit converters. The properties: input signal range $100 \mathrm{mV}-10 \mathrm{~V}$, acquisition frequency 10-100 Hz and internal A/D converter (INT9-1 to 4 channel PCI A/D converter). All physically available analog inputs and outputs as well as virtual channel are automatically monitored and the process values are stored. The process values are transmitted in such a way that the computer screen displays profiles of voltage-time curves. Data acquisition software was used to convert the compressed data form of the history file on the hard disk into text file format. The text files are converted to Excel spreadsheet and the data are then transported into Matlab 2010a for analysis.

\section{The thermistor calibration}

The thermistor used in the experiments had negative temperature coefficient with unknown thermistor constants. The calibrations involved the determination of the thermistor constants and establishing the relationship between the thermistor's resistance and temperature. This was done by fitting both the thermistor and an electronic digital temperature measuring device in a sealed vessel and slowly warming the system until the voltage reached its asymptotic state (steady state). The thermistor was connected to a computer with data acquisition software to provide data of voltage-time real-time plot as shown in Fig. 2. The voltage-time data were used to match the temperature-time data (Fig. 3) obtained from the electronic digital temperature device.

\section{Relationship between resistance and temperature}

Thermistor resistance $\left(R_{\mathrm{TH}}\right)$ and temperature $(T)$ in Kelvin were modeled using the empirical equation developed by Considine [4]:

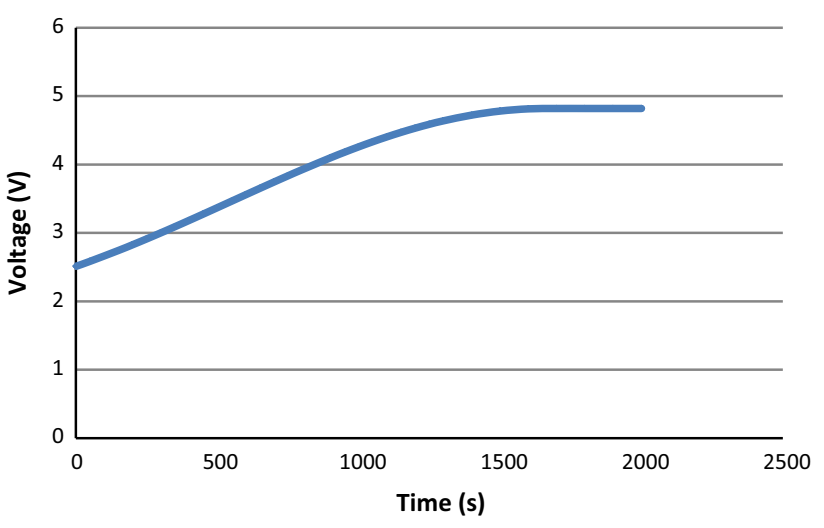

Fig. 2 The thermistor voltage-time profile

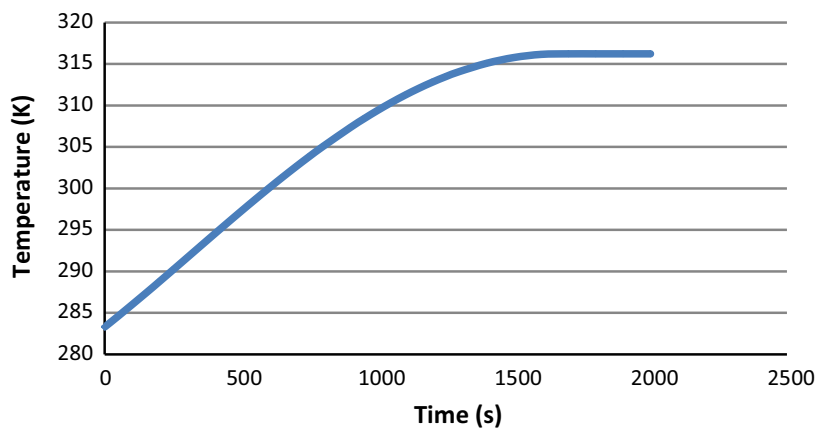

Fig. 3 The thermistor temperature-time profile

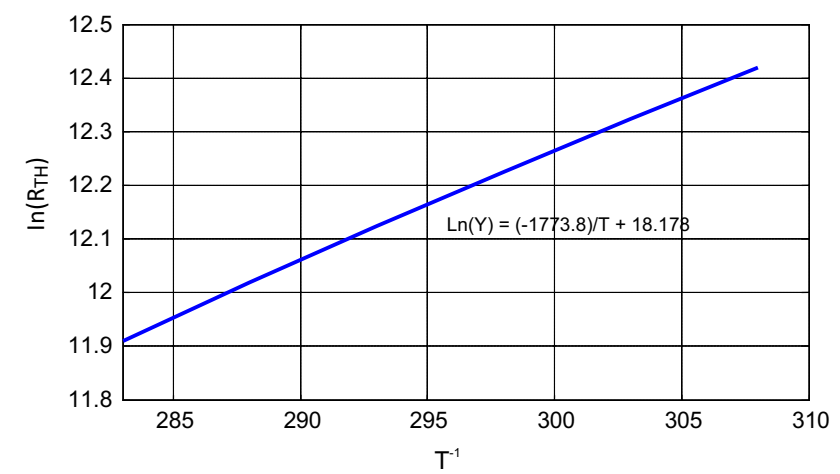

Fig. 4 Regression line of $\ln \left(R_{\mathrm{TH}}\right)$ against $1 / \mathrm{T}$

$R_{\mathrm{TH}}=\exp \left(\frac{B}{T}+C\right)$

or

$\ln \left(R_{\mathrm{TH}}\right)=\frac{B}{T}+C$,

where the parameters $(B)$ and $(C)$ are the thermistor constants and were obtained from experimental calibration using warm water. The constants were determined by 


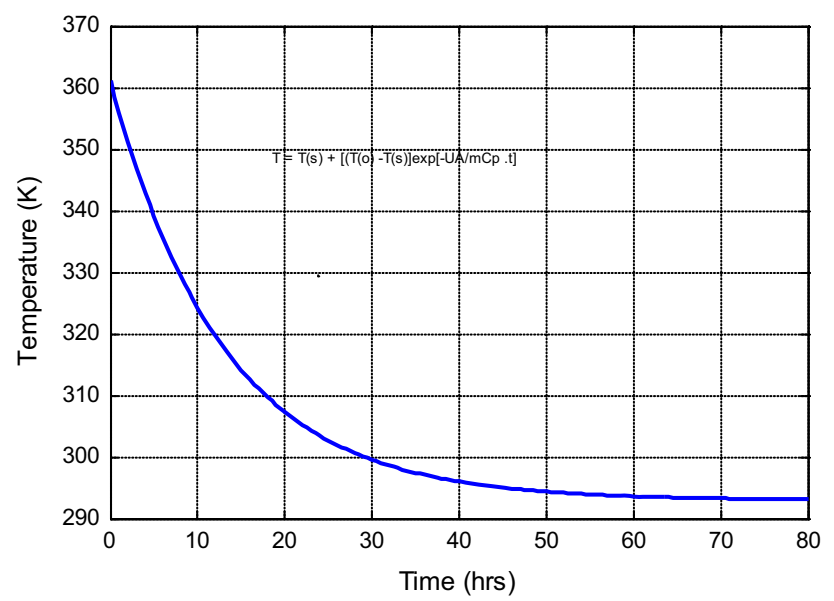

Fig. 5 Thermos flask cooling curve at $T_{(0)}=361 \mathrm{~K}$

fitting the "best" least square straight line plot of $\ln \left(R_{\mathrm{TH}}\right)$ against $1 / \mathrm{T}$, giving the thermistor equation as:

$\ln \left(R_{\mathrm{TH}}\right)=-\frac{1773.8}{T}+18.178$.

The calibration plot is shown in Fig. 4.

Thus for a known current value in the circuit, any measured voltages' resistance $\left(R_{\mathrm{TH}}\right)$ can be calculated by applying Ohm's law and hence the temperature at that time using Eq. (12).

\section{The reactor (thermos flask) calibration}

The reaction vessel used was an ordinary Dewar thermos flask with a removable screw cap lid. The flask had a total volume of $500 \mathrm{~mL}$. The calibration involved the determination of the heat transfer coefficient of the flask and fitting the experimental data to the model described by Eq. (13):

$T=T_{\mathrm{S}}+\left(T_{0}-T_{\mathrm{S}}\right) \exp \left(-\frac{U A}{m C_{\mathrm{P}}} t\right)$.

In this experiment, $400.00 \mathrm{~g}$ of distilled water at $361 \mathrm{~K}$ $\left(T_{0}\right)$ was injected into the reaction vessel, allowing the system temperature to fall over a period of time until the temperature-time profile reached its asymptotic state or the steady-state temperature $\left(T_{\mathrm{S}}\right)$. Figure 5 shows the temperature-time profile of the cooling process.

From Eq. (13), the values of $T_{o}$ and $T_{s}$ were obtained from Fig. 5. Rearrangement of Eq. (13)

$\ln \left(\frac{T-T_{\mathrm{S}}}{T_{0}-T_{\mathrm{S}}}\right)=\ln (Y)=\left(-\frac{U A}{m C_{\mathrm{P}}} t\right)$.

Since $\mathrm{T}$ and $\mathrm{t}$ values are known, least square regression analysis was performed and a straight plot of $\ln (Y)$ against time is shown in Fig. 6.

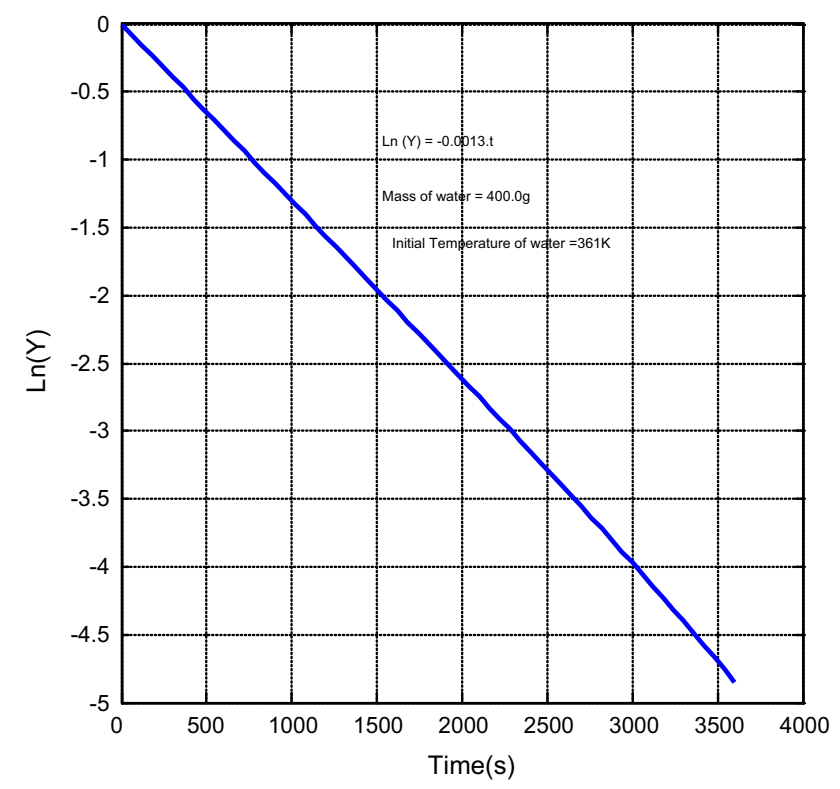

Fig. 6 Regression line of heat transfer coefficient of the reactor

The slope of the straight line of Fig. 6 is given as $0.0013 \mathrm{~s}^{-1}$ which corresponds to the value of the $\mathrm{UA} / \mathrm{mC}_{\mathrm{P}}$ of the flask. This value was used in Eq. 7 above, which was used for the corrections of the experimental temperature values to adiabatic temperature values.

\section{Experimental procedure}

The reactor was filled with a known quantity of one reactant and the system was allowed to reach a steady-state temperature. A known amount of the second reactant was then added. The resulting mixture was then allowed to reach a steady-state temperature before the magnetic stirrer was switched on. The resulting voltage-time data were continuously recorded and converted to temperature-time data and graphs as described in the previous section. The esterification reaction studied was carried out in an ordinary Dewar thermos flask acting as an adiabatic batch reactor of total volume $500 \mathrm{~cm}^{3}\left(0.5 \mathrm{dm}^{3}\right)$ with a magnetic stirrer. Acetic acid/ethanol was the starting material. In all experiments, the mole ratio of acetic acid/ethanol was 1:1, respectively. These quantities (volumes) were used so that at least $60 \%$ of the length of the thermistor probe would be submerged in the liquid mixture. The reactions were carried out at $283 \mathrm{~K}$ and $295 \mathrm{~K}$, respectively.

\section{Results}

The corrected temperature-time curves obtained from Eq. 7 for different basis temperatures for experiments $x$ and 
Fig. 7 a Temperature-time space. b Conversion-time space
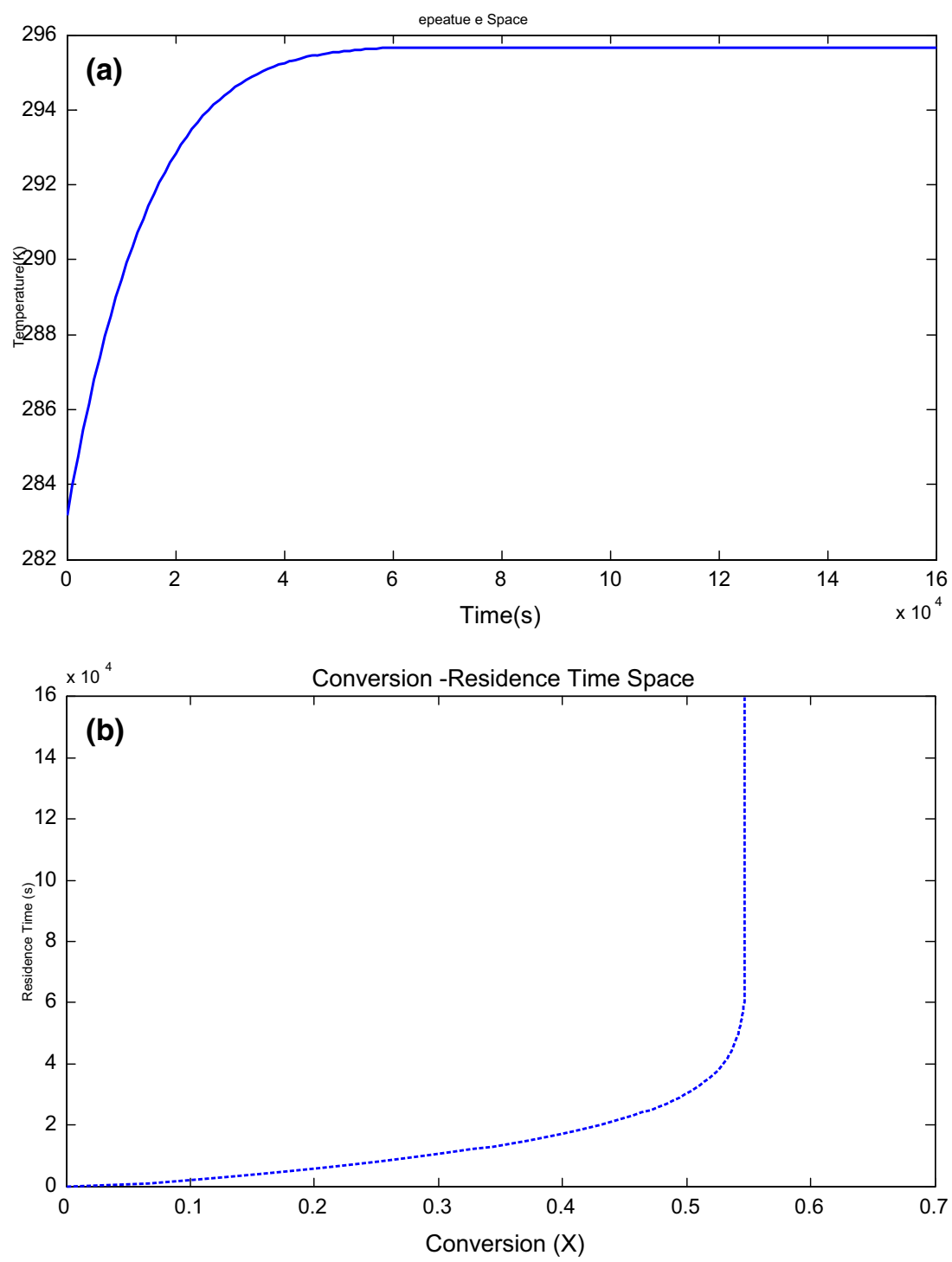

$y$ for the ethyl acetate process are shown in Figs. 7a and 8a. The nature of the curves follows typical s-shaped profiles as expected from any exothermic adiabatic reaction. Figures $7 \mathrm{~b}$ and $8 \mathrm{~b}$ shows the corresponding conversion-time profiles of experiments $x$ and $y$. It is seen that the profiles show the typical adiabatic batch rector profiles as expected. Corrections were made to adjust the experimental data to make the system adiabatic as explained using Eq. (7). A summary of the characteristics of the reaction profiles is shown in Table 1.

\section{Process modeling}

Figure 9 indicates that it is possible to achieve higher conversions than the equilibrium conversion provided by a single adiabatic reactor. The adiabatic line starting from the feed point $(A)$ with feed temperature $283.18 \mathrm{~K}$ and conversion zero reaches equilibrium at point $(x)$ of 0.55 at a maximum temperature of $296.51 \mathrm{~K}$. This point is the maximum achievable conversion for a single adiabatic reactor. From the figure it can be seen that point $(G)$ is the maximum achievable conversion when two reactors are employed.

This is achieved by preheating the feed at point $(A)$ up until one reaches maximum allowable temperature at point $(B)$. Adiabatic reaction is then started at this temperature of $295.59 \mathrm{~K}$ till the reaction reaches equilibrium at point (C) at a temperature of 300.61 and conversion of 0.22 . At this point, interstage cooling is considered. The reaction material at $300.61 \mathrm{~K}$ is cooled down in two steps: first, the cooling process ends at point $(D)$ which is at the same temperature as point $(B)$; then, the second cooling process starts from point $(D)$ and ends at point $(E)$, which is at the 
Fig. 8 a Temperature-time space. b Conversion-time space
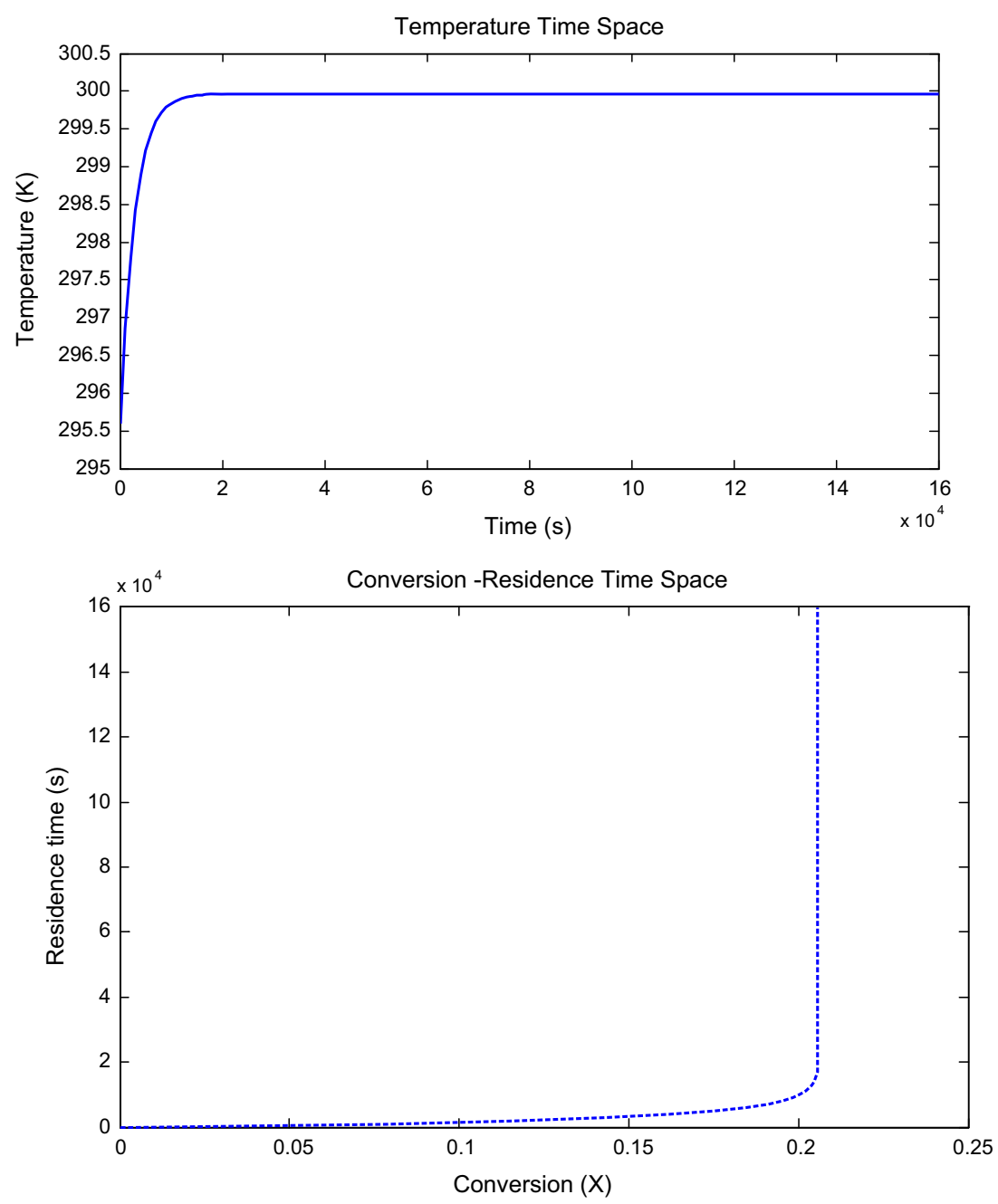

Table 1 Summary of the characteristics of the acetic acid-ethanol reaction

\begin{tabular}{lllcl}
\hline Process & $T_{(0)}(\mathrm{K})$ & $T_{(\max )}(\mathrm{K})$ & $\Delta T_{(\mathrm{ad})}(\mathrm{K})$ & $x_{(\max )}$ \\
\hline$x$ & 283.18 & 296.51 & 13.33 & 0.55 \\
$y$ & 295.59 & 300.61 & 5.02 & 0.21 \\
\hline
\end{tabular}

same temperature as the feed material at point $(A)$. The reaction mixture at this feed point temperature is then reacted adiabatically to the equilibrium point $(F)$ of temperature about $294.8 \mathrm{~K}$ and afterward the reaction material at point $(F)$ is heated up to point $(G)$ of conversion 0.72 as shown in the process modeling Fig. 9. From the geometric point of view, it can be seen that $A B$ and $D E$ have the same length, but in terms of process engineering $A B$ consumes heat while $D E$ gives out heat. Since these lengths represent temperature change, the process implies that energy consumed during process $A B$ should be the same as the energy removed during process $D E$. Since the system (reaction mixture) has a constant heat capacity, the energy balance

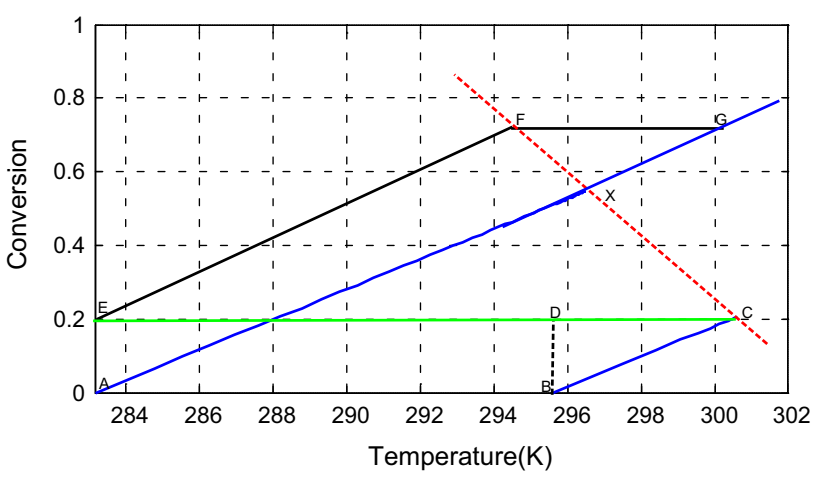

Fig. 9 Crossing the equilibrium line using two adiabatic reactors

for the system is correct. One critical issue here is: how much should one preheat the feed material? Geometrically, it be shown that any optimum configuration only occurs when the temperature at point $(G)$ is the same as that at point $(C)$ [5]. Point $(x)$ has a conversion of 0.55 , while point $(G)$ has a conversion value of 0.73 . It is therefore 


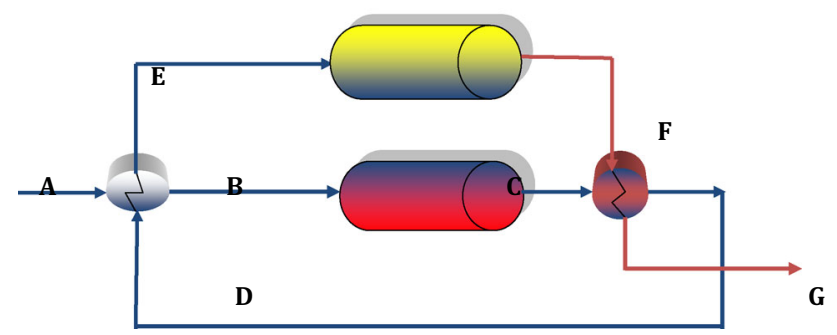

Fig. 10 Optimal reactor configuration for maximum conversion with two reactors

shown that the system results in about $31 \%$ higher conversion than that of a single adiabatic reactor. From the process modeling (Fig. 9), the proposed optimal reactor configuration to achieve this conversion of 0.72 is given in Fig. 10.

\section{Conclusions}

This experimental work has shown that for an exothermic reversible reaction such as the ethyl acetate process, the maximum conversion (equilibrium conversion) for an overall adiabatic system for a two-reactor system with two heat interchanges was about $31 \%$ higher than the equilibrium limit for a single adiabatic batch reactor. It is seen that the optimization did not take cost implications into consideration. Optimizing adiabatic systems by considering cost factors is a more challenging and difficult problem. This result therefore suggests how the optimal configuration with a two-reactor system and two heat interchangers will look like and any of the available process synthesis techniques can be used to perform optimization of such a system by taking cost implication into consideration. The objective of this experimental work is to develop a framework for optimizing exothermic reversible reactions. The results will be very useful if in developing mathematical formulations for optimization, the process engineer must incorporate conversion and cost factors as part of the objective function for the optimization. This experimental result has shown that for a simple adiabatic system with two reactors and two heat interchangers, conversion can be improved significantly with respect to that of a single adiabatic batch reactor.
Acknowledgments N.A. would like to thank the National Research Fund (NRF) of South Africa and the Directors of Center of Material and Process Synthesis Engineering-University of South Africa (UNISA), Johannesburg, South Africa for financial support of this work.

Open Access This article is distributed under the terms of the Creative Commons Attribution License which permits any use, distribution, and reproduction in any medium, provided the original author(s) and the source are credited.

\section{References}

1. Glasser B, Hildebrandt H, Glasser D (1992) Optimal mixing for exothermic reversible reactions. Ind Eng Chem Res 31:154-1549

2. Aries R (1960) The optimal design of adiabatic reactors with several beds. Chem Eng Sci 12:243-252

3. Revalla A, de Lasa L (1987) Cooling exothermic catalytic fixed bed reactors: co-current versus countercurrent operation in methanol conversion reactors. Can J Chem Eng 65:1021-1025

4. Considine DM (1957) Process instruments and controls. Handbook McGraw-Hill Book Co., New York, pp 2-60

5. Nicol W, Hildebrandt D, Glasser D (1998) Crossing reaction equilibrium in an adiabatic reactor system. Dev Chem Eng Mineral Process 6(1/2):41-54

Nana Asiedu holds a B.Sc (Hons) and M.Sc (Chemical Eng.) degrees from KNUST, Kumasi, Ghana. He is currently completing a PhD programme in Chemical Engineering in the University of Witwatersrand Johannesburg, South Africa. He carried out the experimental work and helped in the analysis of the results and drafting of the manuscript.

Diane Hildebrandt holds a PhD in Chemical Engineering from the University of the Witwatersrand Johannesburg, South Africa. She is a full Professor in Chemical Engineering and a Co-Director of MaPs (Material and process synthesis) Engineering, a research center in the Department of Chemical Engineering, University of South Africa (UNISA). She carried out the interpretation of the results and helped in the experimental work.

David Glasser holds a $\mathrm{PhD}$ in Chemical Engineering from University of London (UK). He is a full Professor in Chemical Engineering and the Director of MaPs MaPs (Material and process synthesis) Engineering, a research center in the Department of Chemical Engineering, University of South Africa (UNISA). He conceptualized this work and helped in the analysis of the results and the modeling of the optimal reactor structure. 